Editorials

\title{
Editorial Commentary: Challenge of Non-AIDS-Defining Cancers (NADCs), Focusing on Trans-Activation Response Element (TAR) RNA Embedding Exosomes
}

\author{
Lechuang Chen and "Ge Jin \\ Department of Biological Sciences, Case Western Reserve University School of Dental Medicine, Cleveland, OH 44106, USA
}

Globally, 37.9 million people were living with HIV at the end of 2018 (Organization, 2018). Cancer is a major cause of mortality and morbidity in AIDS patients and chronically HIV-infected people. With the aid of combination Antiretroviral Therapy (ART), people living with HIV (PLWH) have a longer life span. However, because the virus is not completely eradicated, PLWH under antiretroviral therapy may have an increased risk of Non-AIDS-Defining Cancers (NADCs) (Deeks et al., 2013). As defined by NCI, NADCs include Hodgkin lymphoma and cancers of the mouth, throat, liver, lung and anus. Besides HIV infection, other factors, such as older age, infection with other viruses and heavy alcohol or tobacco use, may also increase the risk of developing an NADC (Institute, 0000). Patients with HIV/AIDS are reported to have a two to four-fold increase in risk for head and neck cancers (Powles et al., 2009). Compared with uninfected subjects, the risk of HIV-infected patients with lung cancer has several folds increased (Chaturvedi et al., 2007). Multiple mechanisms contribute to this phenomenon, including longer life expectancy, control of oncogenic infections and exposure to carcinogens (Wang et al., 2014). However, the underlying mechanism for development and progression of certain NADCs remains obscure.

A recent article published in Nat Commun. (Nov 2, 2018), entitled "Exosomes derived from HIV-1-infected cells promote growth and progression of cancer via HIV TAR RNA", Jin's group exhibited an effort to meet this challenge (Chen et al., 2018). This research enrolled 18 HIV-positive patients and discovered how two aggressive cancers used exosomes excreted from HIVinfected $\mathrm{T}$ cells to promote their cancer aggressiveness. Three key messages are present in this study based on the results: First, HIV-infected patients even under ART treatment contain circulating pro-tumor exosomes, in which HIV-specific exosome cargo components are able to promote proliferation, migration and invasion and induce the expression of proto-oncogenes in Head and Neck Squamous Cell Carcinoma (HNSCC) and lung cancer cells in vitro and stimulate xenograft tumor growth in vivo. Second, the HIV Transactivation
Response (TAR) element RNA is found to be overwhelmingly produced in exosomes from HIVinfected patient sera. TAR is a precursor of several HIVencoded miRNAs that forms a stem-loop folding structure in the nascent transcript and facilitates binding of the viral transcriptional Trans-activator (Tat) protein to enhance transcription initiation and elongation of HIV. Most importantly, the HIV TAR RNA in HIVinfected T-cell exosomes is responsible for the expression of the proto-oncogene FOS and TLR3associated induction of Interferon-Stimulated Genes (ISGs) in cancer cells, depending on the loop/bulge region of the molecule. Finally, a nucleotide aptamer called R06, which forms a face-to-face "kissing" structure to block the function of TAR RNA, attenuates gene expression and inhibits progressive behaviors of cancer cells when transfected into HIV-infected T-cell exosomes (Beaurain et al., 2003). This is the first report addressing whether HIV-infected cells are involved in the development and progression of NADCs and identified the critical role of the loop/bulge region of TAR in the pathological event, although further investigation of the TAR RNA in the HIV/AIDS infection and transmission is needed.

Exosomes are a type of extracellular vesicles about 30$120 \mathrm{~nm}$ in diameter released in body fluids by almost all types of cells (Akers et al., 2013). There are various proteins and RNA in exosomes (Vlassov et al., 2012). Interestingly, the data from the analysis by Mass Spectrometry showed that, compared to exosomes from uninfected T-cells, exosomes from HIV-1-infected T-cells are enriched in histones, RNA-binding proteins, Cdks and Src family kinases (Barclay et al., 2019). Consistent with the observation in Jin's group on HNSCC and lung cancer, it has been found that exosomes isolated from HIV-1-infected cells promote the growth and progression of cervical cancer as well (Li et al., 2019). In this study, miR-155-5p was identified as the culprit molecule in exosomes to promote the pathogenic progress of HIV-1associated cervical cancer.

TAR RNA, which has a highly folded stem-bulgeloop structure, plays an important role in the molecular 
interaction between a virus and its host (Bannwarth and Gatignol, 2005). Abundance of TAR RNA exists up to $63 \%$ in exosomes from HIV-1-infected patients (Hladnik et al., 2018), suggesting the importance of the molecule in the pathogenic event. Thus, designing small molecule inhibitors or mimetic peptides to block the function of TAR RNA may be clinically significant in treating the patients. Alternatively, the CRISPRCas9 method can be employed to abolish TAR RNA in exosomes (Sharma, 2019). In addition, development of a new assessment method for TAR RNA in HIV associated cancer patients' biopsies is urgently needed (Sharma, 2019).

Based on updated "Guidelines for Cancer in People Living with HIV" in 2019, PLWH should be offered the same cancer therapies as HIV-negative individuals (Reid et al., 2018). In the US, the life expectancy for most PLWH on ART now approaches that of the general population (Ray et al., 2010). Cancer is the leading cause of death among this population in developed countries (Morlat et al., 2014). In developing countries, NADCs are common among long-term surviving HIV-infected patients not requiring therapy or without access to therapy (Pantanowitz and Dezube, 2009). Half of Asian NADC patients were aged 40-59 years and had advanced-stage disease at diagnosis (Nagata et al., 2018). The proportion of PLWH who are age 65 or older is expected to triple by 2030; it is likely to change the lanscape of both ADCs and NADCs in this population (Shiels et al., 2018). Apparently, early detection and effective treatment of oncogenic viruses may be the best way to prevent the incidence of cancers among PLWH.

\section{References}

Akers, J.C., D. Gonda, R. Kim, B.S. Carter and C.C. Chen, 2013. Biogenesis of Extracellular Vesicles (EV): Exosomes, microvesicles, retrovirus-like vesicles and apoptotic bodies. J. Neuro-Oncol., 113: 1-11. DOI: 10.1007/s11060-013-1084-8

Bannwarth, S. and A. Gatignol, 2005. HIV-1 TAR RNA: The target of molecular interactions between the virus and its host. Curr. HIV Res., 3: 61-71. DOI: 10.2174/1570162052772924

Barclay, R.A., P. Khatkar, G. Mensah, C. DeMarino and J.S.C. Chu et al., 2019. An omics approach to extracellular vesicles from HIV-1 Infected Cells. Cells, 8: 787-787. DOI: 10.3390/cells8080787

Beaurain, F., C. Di Primo, J.J. Toulmé and M. Laguerre, 2003. Molecular dynamics reveals the stabilizing role of loop closing residues in kissing interactions: Comparison between TAR-TAR* and TARaptamer. Nucleic Acids Res., 31: 4275-84. DOI: $10.1093 / \mathrm{nar} / \mathrm{gkg} 467$
Chaturvedi, A.K., R. Pfeiffer, L. Chang, J. Goedert and R. Biggar et al., 2007. Elevated risk of lung cancer among people with AIDS. Aids, 21: 207-213. DOI: 10.1097/QAD.0b013e3280118fca

Chen, L.C., Z. Feng, H. Yue, D. Bazdar and U. Mbonye et al., 2018. Exosomes derived from HIV-1-infected cells promote growth and progression of cancer via HIV TAR RNA. Nature Commun. DOI: 10.1038/s41467-018-07006-2

Deeks, S.G., S.R. Lewin and D.V. Havlir, 2013. The end of AIDS: HIV infection as a chronic disease. Lancet, 382: $1525-1533$. DOI: $10.1016 / \mathrm{S} 0140-6736(13) 61809-7$

Hladnik, A., J. Ferdin, K. Goricar, S.G. Deeks and B.M. Peterlin et al., 2018. Trans-activation response element RNA is detectable in the plasma of a subset of aviremic HIV-1-infected patients (2017, 64: 530-536). Acta Chimica Slovenica, 65: 481-481.

Institute, N.C. NCI dictionary of cancer terms. National Institutes of Health.

Li, H., X. Chi, R. Li, J. Ouyang and Y. Chen, 2019. HIV-1-infected cell-derived exosomes promote the growth and progression of cervical cancer. Int. J. Biol. Sci., 15: 2438-2447. DOI: $10.7150 /$ ijbs.38146

Morlat, P., C. Roussillon, S. Henard, D. Salmon and F. Bonnet et al., 2014. Causes of death among HIVinfected patients in France in 2010 (national survey): Trends since 2000. Aids, 28: 1181-1191. DOI: 10.1097/QAD.0000000000000222

Nagata, N., T. Nishijima, R. Niikura, T. Yokoyama and Y. Matsushita et al., 2018. Increased risk of nonAIDS-defining cancers in Asian HIV-infected patients: a long-term cohort study. BMC Cancer, 18: 1066-1066. DOI: 10.1186/s12885-018-4963-8

Organization, W.H., 2018. Global Health Observatory (GHO) data.

Pantanowitz, L. and B.J. Dezube, 2009. Evolving spectrum and incidence of non-AIDS-defining malignancies. Curr. Opin. Hiv Aids, 4: 27-34.

DOI: 10.1097/COH.0b013e32831a7259

Powles, T., D. Robinson, J. Stebbing, J. Shamash and M. Nelson et al., 2009. Highly active antiretroviral therapy and the incidence of non-AIDS-defining cancers in people with HIV infection. J. Clin. Oncol., 27: 884-890.

DOI: 10.1200/JCO.2008.19.6626

Ray, M., R. Logan, J.A. Sterne, S. Hernández-Díaz and J.M. Robins et al., 2010. The effect of combined antiretroviral therapy on the overall mortality of HIV-infected individuals. Aids, 24: 123-137.

DOI: 10.1097/QAD.0b013e3283324283 
Reid, E., G. Suneja, R.F. Ambinder, K. Ard and R. Baiocchi et al., 2018. Cancer in people living with HIV, version 1.2018 clinical practice guidelines in oncology. J. Nat. Comprehensive Cancer Netw., 16: 986-1017.

Sharma, N.K., 2019. Exosomal packaging of TransActivation Response element (TAR) RNA by HIV-1 infected cells: a pro-malignancy message delivery to cancer cells. Mol. Biol. Rep., 46: 3607-3612.

DOI: $10.1007 / \mathrm{s} 11033-019-04770-2$

Shiels, M.S., J.Y. Islam, P.S. Rosenberg, H.I. Hall and E. Jacobson et al., 2018. Projected cancer incidence rates and burden of incident cancer cases in HIVinfected adults in the United States through 2030. Annals Internal Med., 168: 866-873.

DOI: 10.7326/M17-2499
Vlassov, A.V., S. Magdaleno, R. Setterquist and R. Conrad, 2012. Exosomes: Current knowledge of their composition, biological functions and diagnostic and therapeutic potentials. Biochimica Et Biophysica Acta-General Subjects, 1820: 940-948. DOI: 10.1016/j.bbagen.2012.03.017

Wang, C.C.J., M.J. Silverberg and D.I. Abrams, 2014. Non-AIDS-defining malignancies in the HIVinfected population. Curr. Infect. Dis. Rep., 16: 406-406. DOI: 10.1007/s11908-014-0406-0 